[5] M. T. Sode Mogensen. Medd. Statens Mejeriforsok, 1947, 21.

[6] S. J. Rowland. J. Dairy Res., 1938, 9, 42.

[7] C. Alais. C. R. XIV e Cong. Int. Lait., Rome, 1956, 2, 823.

[8] G. Hurel et G. Mocquot. Le Lait, 1947, 27, 131.

[9] C. W. RaAdsveld. Neth. Milk and Dairy J., 1952, 6, 342.

[10] M. H. Peterson, M. J. Johnson et W. V. Price. J. Dairy Sci., 1948, 31, 55 .

[11] C. W. RaAdsveld et H. Mulder. Neth. Milk and Dairy J., 1949, 3, 222.

[12] G. Mocquot, S. Savole et J. Claveau. C. R. Ac. Agr., 1947, 33, 163.

[13] M. T. Sode Mogensen. Journées Fromagères de Poligny, 1952.

[14] C. Alais, G. Mocquot, H. Nitschmann et P. Zahler. Helv. Chem. Acta, 1953, 36, 1955.

[15] J. Garnier. Ann. Techn. Agr., I.N.R.A., 1957, 3, 245.

[16] I. R. SHerwood. J. Dairy Res., 1935, 6, 204 et 407.

[17] D. G. Vakaleris, N. F. Olson, W. V. Price et S. G. Knight. J. Dairy Sci, $1960,43,1058$.

[18] L. Lindet et L. Ammann. Ann. Inst. Nat. Agr., 1904, 222.

[19] A. W. Bosworth. N. Y. Agr. Exp. Sta. (Geneva). Techn. Bull., 1907, 5.

\title{
REVUE
}

\section{LE LAIT DANS LE MONDE}

par

G. GENIN

Ingénieur E. P. C. I,

\section{GÉNÉRALITÉS}

\section{La production du beurre dans le monde}

D'après les statistiques complètes pour l'année 1961 et le début de 1962, établies par l'Organisation mondiale de l'agriculture, on a enregistré une augmentation sensible de la production de beurre en 1961 au Japon et en Afrique du sud (plus de 14 p. 100) et une augmentation qui varie de 4 à 9 p. 100 en Australie, au Canada en Belgique, en Irlande, en Allemagne de l'ouest, en France et aux Etats-Unis. Mais c'est surtout en Angleterre, pays traditionnellement important importateur de beurre, que l'augmentation a été la plus sensible, puisqu'elle atteint $30 \mathrm{p} .100$, la production passant de 38000 à 49000 tonnes. Il en est résulté que la part revenant à la production nationale dans la consommation du Royaume-Uni est passée de 8 p. 100 en 1960 à 10 p. 100 en 1961. 


\section{Un dictionnaire international de laiterie}

Dès 1955, l'International Dairy Federation avait reconnu la nécessité de préparer un dictionnaire international des termes couramment utilisés en laiterie, afin de faciliter les réunions internationales et un Comité spécialement désigné à cet effet avait été chargé d'établir une liste de termes techniques avec leur traduction en anglais, en français et en allemand. Cependant, ce document était loin d'être complet et en 1958, la même Organisation chargea un Comité de spécialistes d'établir un dictionnaire plus complet, les travaux de ce Comité étant placés sous la direction du Professeur Casalis de France, du Professeur Schulz d'Allemagne et de Mr E. MANN du Commonwealth Bureau of Dairy Science and Technology. La première réunion de ce Comité se tint à Paris en janvier 1959, et on envisage prochainement la publication du dictionnaire, sous le patronage de l'International Dairy Federation; son impression sera assurée par United Trade Press Ltd qui publie la revue britannique bien connue Dairy Industries.

\section{L'utilisation des surplus de lait}

Un groupe d'experts s'est réuni récemment afin d'examiner, sous l'égide de la Food and Agriculture Organisation, les problèmes que pose l'utilisation de l'excès de production mondiale de produits laitiers et de trouver les moyens d'établir de meilleurs échanges de ces produits entre les pays producteurs et les pays consommateurs.

On estime que si la tendance actuelle dans la production des produits laiters se poursuit, des excédents de production ne feront que s'accroître au cours de la prochaine décade, ce qui entraînera une chute des cours mondiaux et obligera les gouvernements à accorder des subsides aux agriculteurs. Le secrétariat de l'Organisation a envisagé différentes mesures afin de lutter contre cette situation et qui devrait conduire à un développement du commerce mondial, à une augmentation de la consommation dans les pays en voie de développement et même, le cas échéant, à une restriction volontaire de la production laitière.

\section{GRANDE-BRETAGNE}

\section{Fabrication du fromage dans des installations continues}

Des recherches se poursuivent activement sur les procédés permettant de réaliser la fabrication continue du fromage. Dans le rapport annuel du National Institute for Research in Dairying, des 
indications ont été publiées sur une nouvelle méthode de traitement du lait qui permet de provoquer la formation de la caillebotte en un temps extrêmement réduit. Un appareil de laboratoire a été construit pour l'étude de ce procédé, qui a fonctionné d'une façon continue pendant une période de deux ans, produisant de la caillebotte à raison de $1 \mathrm{~kg}$ environ à l'heure. Malgré la faible importance de cette production, découlant de la dimension de l'appareil, on a pu produire un fromage de qualité convenable.

D'autre part, le même Institut a également présenté une communication sur un nouveau procédé de chéddarisation en continu, dans lequel le problème de l'égouttage de la caillebotte, qu'il aurait été impossible de résoudre dans une installation capable de traiter $1500 \mathrm{~kg}$ à l'heure de caillebotte, a pu être résolu par l'utilisation de cylindres de capacité de traitement plus faible. L'appareil de cheddarisation proprement dit exécute toutes les opérations mécaniques qui doivent s'effectuer une fois la caillebotte séparée du petit lait et alors que, dans une usine disposant de 12 cuves de précipitation; le traitement exigeait une dépense de main-d'œuvre correspondant à 48 heures d'ouvriers, la nouvelle installation automatique à réduit ce nombre de 6 fois. Le fromage fabriqué dans l'installation a été jugé de qualité sélect ou extra-select.

\section{Pulvérisation du sel sur les fromages par un procédé électro- statique}

Un procédé qui a été déjà employé avec succès dans une fabrique française de camembert, qui procède deux fois par jour à la pulvérisation de sel sur 30000 camemberts, va être également utilisé en Angleterre. On emploie dans ce but un dispositif électrostatique, dénommé Stajet, qui permet de projeter sur les fromages un nuage de particules de sel chargées électriquement, les fromages eux-mêmes portant des charges de signe opposé. Il en résulte que le sel se dépose sur toute la surface des fromages et reste en position pendant un temps suffisant, parfois même pendant plusieurs jours. La différence de potentiel de 90000 volts nécessaire à la projection électrostatique du sel pulvérisé est fournie par un générateur Sames alimenté en courant industriel ordinaire.

\section{Emploi des feuilles thermoplastiques pour l'emballage des produits laitiers}

La Saro Products Ltd livre, sous la référence SP 61, des feuilles plastiques d'une épaisseur de 0,125 à $0,5 \mathrm{~mm}$, exécutées en polystyrène ou en polyéthylène et qui peuvent être utilisées pour l'embal- 
lage de différents produits laitiers, en particulier du fromage et de la crème. Ces feuilles sont obtenues par une technique de boudinage et peuvent avoir un enduit brillant sur une face et mat sur la face opposée, ou au contraire deux enduits mats.

\section{La teneur en strontium 90 du lait}

Le Laboratoire Radiobiologique de l'Agricultural Research Council de Wantage effectue régulièrement un contrôle de la teneur en strontium-90 et en iode-131 du lait et d'autres aliments consommés dans le pays. Un rapport intérimaire a été publié concernant la teneur en iode pour la période allant de décembre 1961 à avril 1962 , et pour la teneur en strontium dans la période des 6 derniers mois de 1961 .

En ce qui concerne la teneur en iode-131, elle a commencé à s'élever à nouveau après la reprise des essais nucléaires en septembre 1961. En ce qui concerne le strontium, la variation a été moins importante, cependant l'apparition de quantités appréciables de strontium-89 a coincidé également avec la reprise des essais sur les armes nucléaires.

\section{Consommation de beurre}

Au cours de l'année 1961, la consommation de beurre dans le Royaume-Uni s'est élevée régulièrement et a atteint une moyenne de 20 pounds par habitant contre 18,1 en 1960. Ce chiffre est le plus élevé atteint depuis 1939. Devant la diminution des cours du beurre, le Butter Information Council a d'ailleurs fait un important effort de propagande, par l'intermédiaire de la télévision et de la presse, afin d'encourager la consommation de ce produit.

\section{Inauguration d'une installation automatique pour la fabrication de la crème glacée}

Les Northern Dairies Ltd de Hull ont inauguré, il y a quelques mois, une installation entièrement automatique pour la fabrication de la crème glacée, installation conduite par un personnel de 4 hommes seulement, non compris l'atelier d'emballage.

Tous les constituants essentiels sont reçus sous la forme liquide et placés dans des réservoirs dont la température est contrôlée à la valeur voulue. Des pompes volumétriques dirigent chacun des constituants dans une cuve de mélangeage et le mélange est ensuite soumis à une pasteurisation à très haute température de construction A. P. V. Les modifications physiques subies par les protéines 
au cours de ce traitement auraient une influence favorable sur la stabilisation du mélange, de sorte qu'il a pu être possible de réduire la quantité de stabilisant, tout en améliorant la qualité et la saveur du produit. Après pasteurisation, le mélange est homogénéisé puis congelé et après un certain vieillissement, il est dirigé dans des appareils de conditionnement et livré en saes de polythène d'une capacité de 4 litres. Toute l'installation a été exécutée en acier inoxydable, son nettoyage se fait sans démontage des appareils.

\section{Production du fromage}

En 1961, la production britannique de fromage s'est élevée à 112000 tonnes et a représenté 46,6 p. 100 des besoins. Le reste a été couvert par des importations en provenance du Commonwealth (41,5 p. 100), du Danemark et de la Norvège (5,3 p. 100) des PaysBas (3,3 p. 100) de Suisse, de France et d'Italie (0,4 p. 100) de la République Irlandaise (0,8 p. 100) et de diverses sources.

Un des problèmes qu'a eu à résoudre l'industrie fromagère a été de développer ses installations pour traiter les quantités de plus en plus importante de lait qu'elle reçoit. Elle a développé dans ce but ses installations automatiques et en général, pour obtenir un produit de qualité plus uniforme, le lait, dès son arrivée, subit un traitement thermique afin de le pasteuriser.

\section{PAYS-BAS}

\section{Installation d'aération du lait}

Une importante laiterie hollandaise, pour effectuer l'aération du lait contenu dans les cuves de traitement, a abandonné le procédé reposant sur l'emploi de compresseurs pour utiliser des aspirateurs d'un modèle analogue à celui qui équipe en modèle réduit les aspirateurs domestiques.

Un type de cette machine, tournant à 8000 tours par minute et commandé par un moteur de $3 \mathrm{CV}$, permet par exemple d'aérer le lait contenu dans 5 cuves ayant chacune une capacité de $16000 \mathrm{l}$. L'installation a été en fonctionnement continu depuis $\mathbf{9}$ mois et a donné entière satisfaction et la même laiterie envisage d'utiliser des appareils analogues tournant à 7000 tours/minute, commandés par des moteurs de $6 \mathrm{CV}$ et capables d'assurer l'aération de 3 cuves d'une capacité de 370001 chacune. 


\section{Développement de la production laitière}

D'après des statistiques complètes, la production laitière en Hollande a atteint en 1961 une production record avec $6953000 \mathrm{t}$ contre 6838000 en 1960. La production de fromage, de lait condensé et de crème a été également supérieure, mais on enregistre une réduction de la production de beurre et de lait en poudre. Voici d'ailleurs les chiffres de production pour 1960 et 1961 en milliers de tonnes :

\begin{tabular}{|c|c|c|}
\hline & 1960 & 1961 \\
\hline Production laitière totale $\ldots \ldots \ldots \ldots \ldots \ldots$ & 6838 & 6953 \\
\hline Lait livré à la consommation $\ldots \ldots \ldots \ldots \ldots$ & 1441 & 1431 \\
\hline Teneur en graisse moyenne $\ldots \ldots \ldots \ldots \ldots$ & $3,79 \%$ & $3,81 \%$ \\
\hline Beurre $\ldots \ldots \ldots \ldots \ldots \ldots \ldots \ldots \ldots \ldots$ & 99,4 & 96,6 \\
\hline Fromage $\ldots \ldots \ldots \ldots$. & 201,6 & 212,9 \\
\hline Lait condensé $\ldots \ldots \ldots \ldots \ldots \ldots \ldots \ldots \ldots$ & 384,6 & 408,6 \\
\hline Lait en poudre $\ldots \ldots \ldots \ldots \ldots \ldots \ldots \ldots \ldots$ & 108,8 & 105,2 \\
\hline Crème $\quad \ldots \ldots \ldots \ldots \ldots \ldots \ldots \ldots \ldots \ldots$ & 16,0 & 18,6 \\
\hline
\end{tabular}

D'autre part, le Bureau des statistiques indique que les exportations de produits laitiers ont été inférieures en valeur et en tonnage à celles de 1960. Le développement des exportations de lait condensé en tonnage a été compensé par une diminution du prix de vente, mais pour les exportations de fromage, c'est l'inverse qui s'est produit.

\section{Nettoyage automatique des installations de fromagerie}

La firme hollandaise Ingenieurs Bureau en Apparatenfabriek Ir. Makkinga de Heerenveen, en collaboration avec l'Association coopérative laitière de Frise, a construit une installation entièrement automatique permettant le nettoyage du matériel utilisé dans la fabrication du fromage.

Les cuves par exemple sont transportées par une bande convoyeuse après retournement et passent sur des pulvérisateurs d'eau qui assurent le lavage de ces cuves. Elles sont ensuite retournées et circulent devant une installation qui les alimente en caillebotte. L'eau de rinçage peut être portée à une température convenable, de telle sorte que les cuves sont elles-mêmes réchauffées et garnies de caillebotte à la température voulue. Toute l'installation est commandée électriquement à partir d'un pupitre de commande central. 


\section{ALLEMAGNE}

\section{Production laitière}

Parmi les pays du Marché commun, l'Allemagne occupe la seconde place et sa production augmente régulièrement. D'autre part, comme le nombre de vaches laitières s'élève aussi chaque année, on pense que pour 1962, la production dépassera largement le chiffre de 18 millions de litres atteint en 1961.

La consommation diminue légèrement de la part des usagers domestiques, mais par contre la production de lait condensé a augmenté et près de la moitié de la production laitière est utilisée pour la fabrication de beurre, ce qui ne suffit d'ailleurs pas encore à couvrir les besoins domestiques. 6 p. 100 seulement de la production sont transformés en fromage et 100000 t sont importées chaque année en particulier de Hollande et du Danemark.

De toute façon, les cours des produits importés ne peuvent agir sur les prix nationaux, car des contrôles sont effectués et des droits de douane s'appliquent sur les importations de produits laitiers. D'autre part, les producteurs reçoivent des subsides gouvernementaux et l'industrie laitière bénéficie indirectement des sommes importantes qui sont accordées par le Gouvernement pour l'amélioration de l'agriculture allemande. On estime qu'en 1961, les producteurs ont reçu en paiement du lait une somme supérieure de $12 \mathrm{p}$. 100 à celle qu'ils avaient reçue en 1956 .

\section{Sacs stérilisables pour l'emballage des produits laitiers}

On a annoncé l'apparition sur le marché de nouveaux sacs constitués par une pellicule double de feuille d'aluminium et d'une membrane plastique, qui peut être portée à la température de $123^{\circ} \mathrm{C}$, ce qui permet la stérilisation de leur contenu. Jusqu'à présent, le principal inconvénient des feuilles d'emballage de ce genre provenait de ce qu'elles ne pouvaient pas supporter des températures de cet ordre, par suite du ramolissement de la couche thermoplastique. Cette difficulté a été surmontée par l'emploi de polyéthylène basse pression, dont le point de ramollissement est de l'ordre de $130^{\circ}$.

La nouvelle pellicule d'emballage est donc constituée d'une feuille d'aluminium de $0,015 \mathrm{~mm}$ d'épaisseur, revêtue sur la face au contact du produit à emballer d'une pellicule de polyéthylène. La feuille d'aluminium met le produit emballé à l'abri de la lumière et son pouvoir réfléchissant pour les rayons calorifiques réduit l'échauffement du contenu. On envisage l'emploi de ces emballages pour la livraison du lait condensé. 


\section{SUÈDE}

\section{Evaporateur centrifuge}

Si la centrifugation est déjà utilisée dans de nombreuses opérations de l'industrie laitière, son utilisation pour la concentration de substances sensibles à la chaleur est un fait nouveau et a été résolu par une firme suédoise.

L'appareil est constitué essentiellement d'assiettes coniques empilées sur un axe et chauffées extérieurement par de la vapeur d'eau. Le produit à traiter pénètre axialement à la partie supérieure de l'appareil et se trouve projeté sur les éléments coniques chauffés où il se trouve réparti, sous l'action de la force centrifuge, sous la forme d'une couche très mince. Par suite de la faible épaisseur de cette couche, de la turbulence qui existe dans l'appareil, et de l'élimination rapide de la vapeur d'eau, un échange intense de chaleur se produit et il en résulte une évaporation très rapide. Dans ces conditions, la quantité de vapeur d'eau évaporée par unité de surface est élevée et le produit concentré est recueilli à la périphérie de l'appareil tandis que la vapeur est éliminée par une tuyauterie reliée à un appareil à vide.

L'évaporation s'effectue dans un temps de l'ordre de une seconde et la valeur nutritive du produit n'est pas affectée. On a pu également concentrer par le même appareil des produits contenant des matières solides en suspension comme par exemple du jus d'agrumes.

\section{DANEMARK}

\section{Exportations de produits laitiers}

Environ 95 p. 100 de la production danoise de conserves de produits laitiers sont exportés, principalement en Extrême-Orient, en Amérique du Sud et en Afrique. Ces exportations portent principalement sur le lait en poudre et sur le lait condensé et les fabriques de ces produits sont dispersées sur toute la surface du pays et utilisent une partie importante de la production laitière danoise, qui s'élève à environ $5.000 \mathrm{t}$ par an.

Le principal produit condensé est livré sous la forme de lait condensé sucré ou non sucré, mais également sous la forme de crème. Le lait condensé contient généralement 30 p. 100 d'extrait sec et 8 à 9 p. 100 de graisse ; il est également fabriqué des quantités importantes de lait écrémé condensé et sucré.

La production de lait en poudre s'est développée régulièrement depuis plusieurs années et représente aujourd'hui environ $40000 \mathrm{t}$ 
de poudre de lait entier ou de poudre de lait écrémé. Ce produit est surtout obtenu par atomisation, mais également par le procédé de séchage sur cylindre. La poudre de lait écrémé est principalement utilisée dans le pays comme matière première pour la préparation de produits alimentaires spéciaux et pour l'alimentation des animaux.

Afin de protéger les consommateurs, on envisage la création d'un label de qualité et les différents producteurs, qui sont groupés en coopératives, ont créé un département spécial chargé de la surveillance des usines.

\section{GREेCE}

\section{Développement de l'industrie laitière}

En 1960, le gouvernement grec a mis en vigueur un plan de 5 ans destiné à la création d'établissements de pasteurisation et de fabrication de produits laitiers dans les principaux centres producteurs et dans les centres urbains. Ces établissements seront exploités par des organisations coopératives de producteurs, le gouvernement accordant un subside représentant 30 p. 100 des frais de construction des usines, les 70 p. 100 restants étant fournis par un prêt de la Banque de l'agriculture de Grèce. Deux usines de fabrication de lait condensé sont également en construction.

Le prix du lait à l'achat et à la vente est réglé par le Ministère du Commerce et varie suivant les différentes régions, en fonction des conditions locales. D'importants efforts devront être entrepris pour améliorer la qualité du lait, étant donné les conditions climatiques défavorables et le manque d'installations de réfrigération danis les fermes.

La production laitière et la consommation se sont établies de la façon suivante en 1959 (indications en tonnes).

\begin{tabular}{|c|c|c|c|c|c|c|}
\hline Nature du lait & $\begin{array}{c}\text { Lait } \\
\text { fluide et } \\
\text { yoghourt }\end{array}$ & Beurre & Fromage & Myzithra & $\begin{array}{l}\text { Alimenta- } \\
\text { tion des } \\
\text { animaux }\end{array}$ & $\begin{array}{l}\text { Produc- } \\
\text { tion lai- } \\
\text { tière totale }\end{array}$ \\
\hline Vache & 203000 & 41000 & 22700 & & 67000 & 333700 \\
\hline Bufflesse ...... & 8000 & 11800 & 4300 & & 6400 & 30500 \\
\hline Brebis ........ & 63000 & 10000 & 220000 & 1400 & 63000 & 357400 \\
\hline Chèvre $\ldots \ldots \ldots$ & 66000 & 21000 & 131500 & 2500 & 39800 & 260800 \\
\hline Total . . & 340000 & 83800 & 378500 & 3900 & 176200 & 982400 \\
\hline
\end{tabular}

Les importations de lait condensé en 1959 se sont élevées à 15934 t. Celles de lait écrémé en poudre à 9997 t et celles de lait entier en poudre à 861 t. 


\section{PORTUGAL}

\section{Nouvelle laiterie de Lisbonne}

Récemment, une importante laiterie, capable de traiter 1000001 de lait par jour a été inaugurée à Lisbonne. Cette installation appartient à l'Uniao das Cooperativas Abastecedoras de Leite de Lisboa qui a été fondée par un certain nombre de coopératives laitières, et elle alimente la ville de Lisbonne et son voisinage. Elle fabrique également différents types de produits laitiers tels que fromages, beurre, poudre de lait, lait stérilisé et yoghourt.

La laiterie a été inaugurée par le Ministre portugais de l'Economie, en présence des membres du Gouvernement et des représentants de la profession. L'installation a été conçue par le Bureau coopératif des laiteries suédoises de Stockholm, en collaboration avec la Sociedade Avanco Lda de Lisbonne. Les machines et l'outillage ont été achetés dans différents pays européens.

\section{BIBLIOGRAPHIE ANALYTIQUE}

\section{$1^{e}$ LES LIVRES}

Quittet (E.). - Les races bovines françaises. 1 Vol. rel. 80 pages, (21x27), 52 photos, 26 cartes dont 2 en couleurs en dépliant relié. Editeur : La Maison Rustique, 26, rue Jacob. Paris (6e), 1963. Prix franco rec. 25 F. (C.C.P. Paris 209-39).

Où en sont les races bovines françaises ?

Dans quelle mesure la politique de regroupement du Ministère de l'Agriculture a-t-elle porté ses fruits ?

Quelles sont les races qui bénéficient de l'évolution générale?

Comment se dessine l'avenir?

Autant de questions auxquelles l'ouvrage que nous présentons ici répond grâce aux données d'une enquête récente.

Cet ouvrage comprend trois parties :

LA PREMIÈRe, d'ordre statistique, se rapporte aux effectifs des groupes ethniques. Les données statistiques sont suivies par quelques commentaires pour dégager le sens général de l'évolution. Les constatations faites peuvent, en effet, servir de base à une partie de la politique technique de l'élevage français. L'auteur suggère les grands traits de ce que peut être cette politique. 